\title{
Facial Width-to-Height Ratio as a Cue of Threat: An Initial Event-Related Potential Study
}

\author{
Petri Kajonius ${ }^{1,2, ~ *, ~ H a n s ~ E l d b l o m ~}{ }^{1}$ \\ ${ }^{1}$ Department of Cognitive Neuroscience, University of Skovde, Skovde, Sweden \\ ${ }^{2}$ Department of Behavioural Science, University West, Trollhattan, Sweden
}

Email address:

Petri.kajonius@hv.se (P. Kajonius)

${ }^{*}$ Corresponding author

To cite this article:

Petri Kajonius, Hans Eldblom. Facial Width-to-Height Ratio as a Cue of Threat: An Initial Event-Related Potential Study. International Journal of Psychological and Brain Sciences. Vol. 5, No. 1, 2020, pp. 1-4. doi: 10.11648/j.ijpbs.20200501.11

Received: January 2, 2019; Accepted: December 23, 2019; Published: January 4, 2020

\begin{abstract}
The background for the present study is that facial width-to-height ratio (fWHR) is suggested to function as an evolutionary cue for threatening behavior such as perceived aggression. With a novel approach, in this pilot study, we explored whether fWHR can be detected in observers' brain responses measured by event-related potentials (ERP), specifically, the Late Positive Potential (LPP) component (400-3000ms after stimuli onset). The hypothesis was that faces with a high fWHR would elicit a larger LPP amplitude than faces with a low fWHR. The results showed that faces with high fWHRs were indeed perceived as more aggressive and elicited significantly greater LPP amplitudes. The conclusion lends initial support to fWHR serving as a facial cue with evolutionary relevance. We caution that future full-length studies need to take the current small-scale study's limitations into consideration.
\end{abstract}

Keywords: Facial Width-to-Height Ratio, Aggression, ERP, LPP, fWHR

\section{Introduction}

Research from cognitive neuroscience suggests that the human brain's visual system is particularly sensitive to cues in faces for the benefit of social and emotional interactions [1]. Furthermore, judgments of faces in terms of aggression may carry an evolutionary purpose, such as distinguishing between friend and foe [2]. One cue in particular is the facial width-to-height ratio (fWHR) (See Figure 1). Particularly males with a high fWHR tend to be more aggressive (e.g., socially dominant) [3], and congruent with this, observers perceive faces with a high fWHR as more aggressive [4-7].

\subsection{An ERP Framework for fWHR}

To date, there has to our knowledge not been electroencephalographic (EEG) studies examining brain activity and fWHR. Brain activity can be measured as event-related potentials (ERPs), which is made up of the grand average of many individual EEG trials [8]. In particular, Late Positive Potential (LPP) is an element that indicate attention to emotive inducements and develops at around $400 \mathrm{~ms}$ after stimulus onset in the central-parietal areas of the scalp and last for up to several seconds [9]. It is believed that particularly arousing inducements elicit higher LPPs [10]. The idea for the present study is that if fWHR is detectable by observers and provides a cue of threat [6] then LPP amplitudes will be heightened. Consequently, we test the hypothesis that the LPP will show higher amplitudes in subjects when watching a high fWHR-face contrasted to a low fWHR-face.

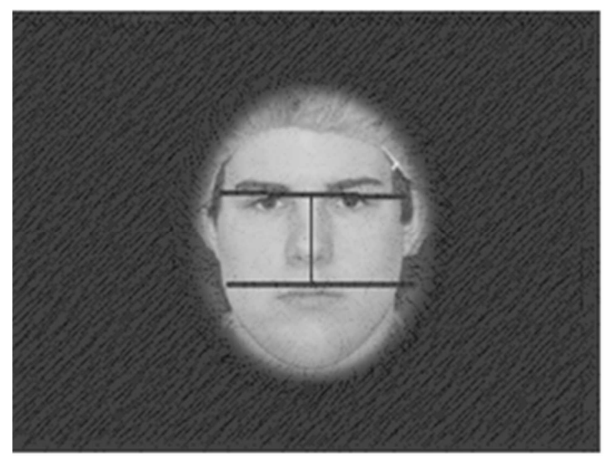




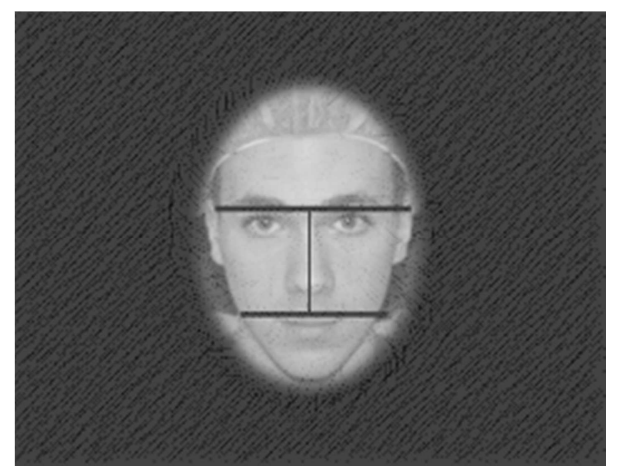

Figure 1. Examples of faces with low and high fWHR: The male to the left has a high $f W H R$. The male on the right has a low fWHR. The black lines show the measurement of $f W H R$.

\subsection{An Evolutionary Framework for fWHR}

One idea behind fWHR is that particularly males develop distinct facial characteristics for mating advantages [11]. These benefits can be intra-sexual, i.e., chasing off competition, as well as inter-sexual, i.e., attracting females. Studies have shown that masculine facial features, particularly in the face, are regarded as socially and physically dominant $[12,13]$. Consequently, evidence puts forth that men with high fWHRs are seen by others as more assertive and intimidating [14]. Perceptual and sensory systems may have developed through evolution to detect threats of aggression $[15,16]$. Another competing line of thinking holds that high fWHRs simply resemble components of anger and are so seen as intimidating [17]. In sum, the overall idea behind fWHR from an evolutionary and biological perspective is that it has evolved as a cueing system of dominance and mating quality $[6,13]$.

\section{Method}

\subsection{Subjects}

27 subjects, 7 females and 20 males, right-handed with normal eyesight, between $18-40$ years of age $(\mathrm{M}=23)$ participated in the study. They stated no record of medical history. All were students recruited voluntarily without compensation at the university and were all fluent in English. Informed consent was obtained from all individual participants included in the study. The use of EEG on subjects was approved by the National Board of Ethics.

\subsection{Procedure and Stimuli}

Subjects were introduced to the EEG-lab and seated in a chair in front of a monitor, placed $115 \mathrm{~cm}$ from the screen. While seated in the EEG-chamber, subjects filled out a questionnaire rating aggressiveness of 24 neutral male faces, varying in fWHRs. The rating made use of a 9-point Likert scale (1- not at all aggressive, 5 - somewhat aggressive, 9 very much aggressive). Immediately after the ratings were completed, the faces were presented on the monitor in randomized order.

The stimuli (faces) were presented on a 24 " screen with a
$1920 \times 1080$ p resolution at a frame rate of $60 \mathrm{~Hz}$. This was created using E-Prime (Version 2.0: Psychology Software Tools, Inc., USA). A sample of 24 neutral faces was retrieved from Cheryl McCormick, Brock University, Canada, which was used in previous fWHR-studies [18]. See Figure 1 for example of what the pictures looked like. The task consisted of 5 blocks with 24 trials/faces in each block, in total 120 stimuli per subject. Each trial lasted for $3000 \mathrm{~ms}$. The time between every trial was $800-1000 \mathrm{~ms}$ where a blank screen was shown.

\subsection{Equipment and Data Filtering}

32 active $\mathrm{AG} / \mathrm{AgCI}$ electrodes were positioned according to the international 10-20 placement system. The ERP was recorded using two active g. GAMMAsys electrode interfaces and two g. USBamp amplifiers (G. Tec Medical Engineering $\mathrm{GmbH}$, Austria). The system transmitted a transformed output signal with an impedance of $1 \mathrm{k} \Omega$. A sampling rate of $512 \mathrm{~Hz}$ with a $0.01 \mathrm{~Hz}$ high-pass filter and a $30 \mathrm{~Hz}$ low-pass filter was used. The data was processed by the EEGLAB toolbox (version 13.6.5b) for MATLAB (R2015a), resampled from $512 \mathrm{~Hz}$ down to $256 \mathrm{~Hz}$ and referenced to the average of the left and right mastoids. Artifact rejection was applied. The data was epoched into segments of $400 \mathrm{~ms}$ to $3000 \mathrm{~ms}$ relative to stimulus onset. The period from $-400 \mathrm{~ms}$ to 0 was set as the baseline period.

\subsection{Analysis of the LPP}

The Late Positive Potential (LPP), which reflects EEG activity due emotional arousal, was hypothesized to be greater when observing threatening faces than non-threatening faces [19]. To test this, we split all the stimuli (faces) into one high fWHR group $(\mathrm{M}=1.97$; $\mathrm{SD}=.09)$ and one low fWHR-group $(\mathrm{M}=1.76 ; \mathrm{SD}=.08)$. A within-group design (dependent t-tests) was implemented, exposing all subjects to all 24 faces, which has the advantage of subjects being their own control for error. We created grand averages of all subjects' individual ERPs based on the latency of interest, $400 \mathrm{~ms}-3000 \mathrm{~ms}$, divided into high and low fWHR conditions. To check whether fWHR might be a cue for perceived threat (aggression), zero-order correlations of the subjects' rating of faces and stimuli-fWHRs was calculated.

\section{Results}

To answer whether the LPP-component would yield higher amplitudes in subjects when observing a face with a high fWHR compared to a face with a low fWHR, grand averages were compared. The within-subjects' LPP-amplitude $(\mu \mathrm{V})$ was substantially greater for faces with high fWHRs $(\mathrm{M}=9.12, \mathrm{SD}$ $=7.23)$ compared to low fWHRs $(\mathrm{M}=3.94, \mathrm{SD}=3.59)$. A paired sample t-test showed that the mean amplitude-differences $(\mu \mathrm{V})$ within subjects when observing faces with high fWHRs compared to faces with low fWHRs were significant $(t=3.13, p=.006)$. Figure 2 illustrates the ERP across the time range of interest. Inspecting the latency for LPP 
amplitudes for high and low fWHRs, differences were most pronounced in the mid and late LPP $(800-3000 \mathrm{~ms})$. Even in the late LPP the differences when observing faces with high fWHRs $(\mathrm{M}=4.87, \mathrm{SD}=4.52)$ compared to low fWHRs $(\mathrm{M}=$ $1.46, \mathrm{SD}=1.91)$ was significant $(\mathrm{t}=3.01, \mathrm{p}=.007)$.

To further explore what the differences in LPP-amplitudes between high and low fWHR could reflect on a psychological level, the questionnaire data was subjected to paired samples t-testing. In confirmation to the idea behind the experiment, subjects rated faces with high fWHRs $(\mathrm{M}=4.20, \mathrm{SD}=2.12)$ as more aggressive than faces with low fWHRs $(\mathrm{M}=2.70, \mathrm{SD}$ $=1.72)(\mathrm{p}<.001)$. A zero-order correlation analysis showed a clear positive correlation between perceived aggression scores and fWHR $(\mathrm{r}=.33, \mathrm{p}<.001)$, indicating that as subjects perceive variations in $\mathrm{fWHR}$, they interpret these as a threatening cue.

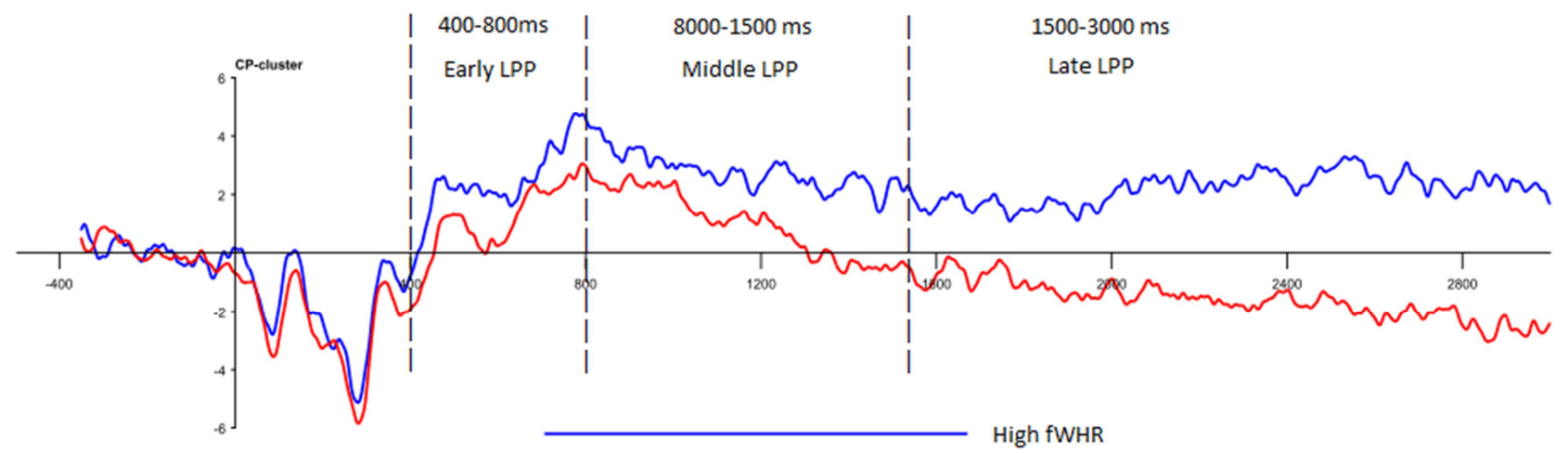

LoW fWHR

Figure 2. Grand average LPP-differences for high (blue amplitudes) vs. low (red amplitudes).

\section{Discussion}

This initial pilot-study's hypothesis that the LPP component would yield higher amplitudes in subjects when observing a face with a high fWHR compared to a face with a low fWHR was confirmed. Furthermore, despite the small sample, the questionnaire data showed that observers do detect variations in fWHR and rate these as signals of aggression. Previous studies have shown that males with high fWHRs behave in slightly more aggressive ways, and more convincingly so, that observers perceive aggression with fWHR. The present study was able to replicate that observers perceive fWHR as aggressive, and in addition for the first time, report that this translates into EEG-signatures. Specifically, the LPP-amplitudes for low fWHRs rapidly decrease towards baseline whereas the amplitudes for high fWHRs remain relatively high throughout the whole LPP. Speculatively, one interpretation is that the fWHR stimulus entails a level of evolutionary significance that evoked sustained attention with the subjects. Conversely, for faces with low fWHRs, attention is only elicited in the first glance.

\subsection{Limitations}

We only had complete data from 19 participants, yielding low statistical power and thus being susceptible to spurious results. In addition, the variance between subjects is usually large in EEG-settings. However, this may have been compensated somewhat by the experimental design, since each subject was exposed to a total of 120 stimuli per subject, making the total number of data-points sufficiently large $(\mathrm{N}=$
2280) [20].

There are also lingering questions about what produced the EEG-amplitude differences, since no competing hypotheses were posed in the present study - Was it mainly an automatic perceptual response based on fWHR or rather a post-perceptual response? Earlier studies such as Carré et al. (2009) primed subjects on aggression when conducting experiments measuring aggression scores based on fHWR. From the present study, we do know that the subjects perceived aggression with fWHR, but we do not know if the LPP activity differences were entirely due to post-processing.

\subsection{Conclusion}

Results from this initial small-scale pilot study showed that subjects yielded higher LPP amplitudes for high fWHR faces, and that subjects rated high fWHR faces as more aggressive. The conclusion leans towards initial support for fWHR serving as a facial cue. Future studies need to take the current small-scale pilot study's limitations into consideration. Nevertheless, there is some credence to the possibility that humans have evolved a specific mechanism to promptly detect a potential threat in male faces.

\section{Acknowledgements}

Thanks to the Department of Cognitive Neuroscience and the EEG-lab for making this study possible. Special thanks to Oskar MacGregor, head of the EEG-lab for solving many improbable situations and making this exploratory novel study possible. On behalf of all authors, the corresponding author states that there is no conflict of interest. 


\section{References}

[1] McGugin, R., \& Gauthier, I. (2013). Face Recognition. In: K. Ochsner, Kosslyn, S. M (Eds.), The Oxford Handbook of Cognitive Neuroscience, Volume 2: The Cutting Edges (p. 165). Oxford University Press.

[2] Rhodes, G. (2006). The evolutionary psychology of facial beauty. Annu. Rev. Psychol., 57, 199-226.

[3] Geniole, S. N., Keyes, A. E., Carré, J. M., \& McCormick, C. M. (2014). Fearless dominance mediates the relationship between the facial width-to-height ratio and willingness to cheat. Personality and Individual Differences, 57, 59-64.

[4] Borgi, M., \& Majolo, B. (2016). Facial width-to-height ratio relates to dominance style in the genus Macaca. PeerJ, 4, e1775.

[5] Deska, J. C., Lloyd, E. P., \& Hugenberg, K. (2018). The face of fear and anger: Facial width-to-height ratio biases recognition of angry and fearful expressions. Emotion, 18 (3), 453-464.

[6] Geniole, S. N., Denson, T. F., Dixson, B. J., Carré, J. M., \& McCormick, C. M. (2015). Evidence from meta-analyses of the facial width-to-height ratio as an evolved cue of threat. PloS one, 10 (7), e0132726.

[7] Lieberz, K. A., Windmann, S., Geniole, S. N., McCormick, C. M., Mueller-Engelmann, M., Gruener, F.,... \& Steil, R. (2017). The facial width-to-height ratio determines interpersonal distance preferences in the observer. Aggressive behavior, 43 (5), 460-470.

[8] Luck, S. (2014). An introduction to the Event-related Potential Technique. New York, NY: MIT press.

[9] Cuthbert, B. N., Schupp, H. T., Bradley, M. M., Birbaumer, N., \& Lang, P. J. (2000). Brain potentials in affective picture processing: Covariation with autonomic arousal and affective report. Biological Psychology, 52 (2), 95-111.

[10] Schupp, H. T., Öhman, A., Junghöfer, M., Weike, A. I., Stockburger, J., \& Hamm, A. O. (2004). The facilitated processing of threatening faces: an ERP analysis. Emotion, 4 (2), 189-200.
[11] Carré, J. M., \& McCormick, C. M. (2008). In your face: facial metrics predict aggressive behaviour in the laboratory and in varsity and professional hockey players. Proceedings of the Royal Society of London B: Biological Sciences, 275 (1651), 2651-2656.

[12] Puts, D. A. (2010). Beauty and the beast: Mechanisms of sexual selection in humans. Evolution and Human Behavior, 31 (3), 157-175.

[13] Puts, D. A., Jones, B. C., \& DeBruine, L. M. (2012). Sexual selection on human faces and voices. Journal of sex research, 49 (2-3), 227-243.

[14] Stirrat, M., Stulp, G., \& Pollet, T. V. (2012). Male facial width is associated with death by contact violence: narrow-faced males are more likely to die from contact violence. Evolution and Human Behavior, 33 (5), 551-556.

[15] Blanchard, D. C., Griebel, G., Pobbe, R., \& Blanchard, R. J. (2011). Risk assessment as an evolved threat detection and analysis process. Neuroscience \& Biobehavioral Reviews, 35 (4), 991-998.279.

[16] Sell, A., Cosmides, L., Tooby, J., Sznycer, D., Von Rueden, C., \& Gurven, M. (2009). Human adaptations for the visual assessment of strength and fighting ability from the body and face. Proceedings of the Royal Society of London B: Biological Sciences, 276 (1656), 575-584.

[17] Eisenbruch, A. B., Lukaszewski, A. W., Simmons, Z. L., Arai, S., \& Roney, J. R. (2017). Why the Wide Face? Androgen Receptor Gene Polymorphism does not Predict Men's Facial Width-to-Height Ratio. Adaptive Human Behavior and Physiology, 4 (2), 1-14.

[18] Carré, J. M., McCormick, C. M., \& Mondloch, C. J. (2009). Facial structure is a reliable cue of aggressive behavior. Psychological Science, 20 (10), 1194-1198.

[19] Hajcak, G., MacNamara, A., \& Olvet, D. M. (2010). Event-related potentials, emotion, and emotion regulation: An integrative review. Developmental Neuropsychology, 35 (2), 129-155.

[20] Schönbrodt, F. D., \& Perugini, M. (2013). At what sample size do correlations stabilize? Journal of Research in Personality, 47 (5), 609-612. 